\title{
Glycaemic index and glycaemic load values of a selection of popular foods consumed in Hong Kong
}

\author{
Kris Y. Lok $^{1}$, Ruth Chan ${ }^{1}$, Dicken Chan ${ }^{2}$, Liz Li $^{1}$, Grace Leung ${ }^{1}$, Jean Woo ${ }^{1}$, Helen J. Lightowler ${ }^{3}$ \\ and C. Jeya K. Henry ${ }^{3}$ \\ ${ }^{1}$ Department of Medicine and Therapeutics, Prince of Wales Hospital, The Chinese University of Hong Kong, Shatin, Hong Kong \\ ${ }^{2}$ Jockey Club Centre for Osteoporosis Care and Control, Prince of Wales Hospital, The Chinese University of Hong Kong, Shatin, \\ Hong Kong \\ ${ }^{3}$ Nutrition and Food Science Group, School of Life Sciences, Oxford Brookes University, Gipsy Lane Campus, Headington, Oxford \\ $O X 3 O B P, U K$
}

(Received 10 September 2008 - Revised 10 August 2009 - Accepted 17 August 2009 - First published online 28 September 2009)

\begin{abstract}
The objective of the present paper is to provide glycaemic index (GI) and glycaemic load (GL) values for a variety of foods that are commonly consumed in Hong Kong and expand on the international GI table of Chinese foods. Fasted healthy subjects were given 50g of available carbohydrate servings of a glucose reference, which was tested twice, and test foods of various brands of noodles $(n 5)$, instant cereals $(n 3)$ and breads ( $n$ 2), which were tested once, on separate occasions. For each test food, tests were repeated in ten healthy subjects. Capillary blood glucose was measured via finger-prick samples in fasting subjects $(-5,0 \mathrm{~min})$ and at 15, 30, 45, 60, 90 and 120 min after the consumption of each test food. The GI of each test food was calculated geometrically by expressing the incremental area under the blood glucose response curve (IAUC) of each test food as a percentage of each subject's average IAUC for the reference food. GL was calculated as the product of the test food's GI and the amount of available carbohydrate in a reference serving size. The majority of GI values of foods tested were medium (a GI value of 56-69) to high (a GI value of 70 or more) and compared well with previously published values. More importantly, our dataset provides GI values of ten foods previously untested and presents values for foods commonly consumed in Hong Kong.
\end{abstract}

Glycaemic index: Glycaemic load: Chinese foods

Hong Kong is similar to other affluent countries with the continued rise in prevalence in obesity and diabetes. A popular concept introduced by Jenkins et al. ${ }^{(1)}$ known as the glycaemic index (GI) has been of potential benefit. A substantial number of epidemiological and interventional studies have reported beneficial associations of lower-GI diets across a wide spectrum of pathophysiological conditions, including diabetes, CVD, obesity and certain forms of cancer ${ }^{(2)}$. The scientific literature also shows that the metabolic effects of low-GI diets acutely induce weight loss, lower fasting glucose and insulin levels, reduce TAG levels and improve blood pressure $^{(3)}$.

The GI is a system for classifying carbohydrate-containing foods according to glycaemic response. GI is defined as the incremental area under the blood glucose response curve (IAUC) after a standard amount of a control food (either white bread or glucose) is consumed ${ }^{(1)}$. The GI of a specific food or meal is determined primarily by the nature of the carbohydrate consumed and by other dietary factors that affect nutrient digestibility or insulin secretion. The GI of foods varies significantly due to factors such as particle size, cooking and food processing, other food components (for example, fat, protein, dietary fibre) and starch structure ${ }^{(4)}$. Consequently, there is considerable variation in the GI of the same food manufactured in different countries. Previous studies on GI, which is widely used and endorsed by the FAO/ $\mathrm{WHO}^{(5)}$, have been conducted mainly in Western countries.

There is persuasive evidence that GI is especially important for individuals with diabetes. High-glycaemic diets have been linked with higher insulin levels and an elevated risk of developing diabetes ${ }^{(6,7)}$. Two mechanisms have been suggested whereby intake of high-GI carbohydrates may increase the risk of type 2 diabetes by overstimulating insulin secretion or contributing to pancreatic $\beta$-cell dysfunction which can result in impaired glucose tolerance and eventually type 2 diabetes ${ }^{(7,8)}$.

Publication of international GI values is needed for various ethnic food groups to ensure a comprehensive list is available to utilise in practice. A recent review of GI values published to date lists 2487 different items across a range of globally produced food groups and brands ${ }^{(9)}$. However, the vast majority of published GI values are Australasian, British or Canadian in origin, with some Danish, French and Swedish values. There are currently few published GI values for Chinese

Abbreviations: GI, glycaemic index; GL, glycaemic load; IAUC, incremental area under the blood glucose response curve.

*Corresponding author: Kris Yuet Wan Lok, fax +852 2602 3500, email kris.lok@cuhk.edu.hk 
foods. The aim of the present study is to provide reliable values of GI of some common foods consumed in Hong Kong including Chinese foods.

\section{Experimental methods}

Subjects

A total of twenty-three healthy subjects (three male and twenty female) were recruited to take part in the study. The subject characteristics are shown in Table 1. Subjects were recruited through email posters distributed at The Chinese University of Hong Kong, the same methodology previously used by UK researchers ${ }^{(10)}$. Exclusion criteria were as follows: age $<18$ or $>40$ years; $B M I \geq 23 \mathrm{~kg} / \mathrm{m}^{2}$; fasting blood glucose value $>6.1 \mathrm{mmol} / \mathrm{l}$; and with known history of diabetes or impaired glucose tolerance. Ethical approval for the study was obtained from the respective university's research ethics committee. Subjects were given full details of the study protocol and the opportunity to ask questions. All subjects gave written informed consent before participation.

The present study was conducted according to the guidelines laid down in the Declaration of Helsinki and all procedures involving human subjects were approved by the Chinese University of Hong Kong, Clinical Research Ethics Committee (CRE-2007.493-T).

All anthropometric measurements were made in the fasting state. Height was recorded to the nearest $1 \mathrm{~cm}$ using a physician beam measure (Seca 220; Seca, Hamburg, Germany) with subjects wearing no shoes. Body weight was recorded to the nearest $0.1 \mathrm{~kg}$ using the Seca 220, wearing light clothing and no shoes. BMI was calculated and body fat percentage was measured using a Tanita analyser (TBF-410; Tanita, Tokyo, Japan). Characteristics of the subjects are shown in Table 1.

\section{Study protocol}

The protocol used was adapted from that described by Wolever et al. ${ }^{(11)}$ and Brouns et al. ${ }^{(12)}$, and is in line with procedures recommended by the $\mathrm{FAO} / \mathrm{WHO}^{(5)}$. For each test food, tests were repeated in ten subjects. On the day before a test, subjects were asked to restrict their intake of alcoholand caffeine-containing drinks and to restrict their participation in intense physical activity. Subjects were also told not to eat or drink after 21.00 hours the night before a test, although water was allowed in moderation.

Table 1. Subject characteristics

(Mean values and standard deviations for twenty-three subjects)

\begin{tabular}{lcl}
\hline & Mean & SD \\
\hline Age (years) & 25.1 & 4.6 \\
Height $(\mathrm{m})$ & 1.60 & 0.07 \\
Weight $(\mathrm{kg})$ & 50.0 & 5.9 \\
BMl $\left(\mathrm{kg} / \mathrm{m}^{2}\right)$ & 19.4 & 1.6 \\
Body fat $(\%)$ & 23.4 & 4.7 \\
Waist circumference $(\mathrm{cm})$ & 73.2 & 6.7 \\
Hip circumference $(\mathrm{cm})$ & 91.0 & 6.3 \\
Fasting blood glucose $(\mathrm{mmol} / \mathrm{l})$ & 4.81 & 0.59 \\
\hline
\end{tabular}

\section{Test foods}

A number of different foods were tested, including breads, cereals and noodles. These foods represent a diverse range of foods commonly consumed in Hong Kong. All foods were tested in equivalent available carbohydrate amounts $(50 \mathrm{~g})$ and compared with a reference food (glucose). Available carbohydrate and fibre values were provided by the manufacturers. Table 2 lists the composition of the test foods. All foods were tested immediately after cooking. Foods were bought and prepared on the day of testing following the manufacturers' instructions.

In accordance with $\mathrm{FAO} / \mathrm{WHO}$ recommendations, subjects tested each test food once and the reference food twice in random order on separate days, with at least a $1 \mathrm{~d}$ gap between measurements to minimise carry-over effects. Subjects were studied in the morning after a $12 \mathrm{~h}$ overnight fast. Subjects consumed the reference or test foods at a comfortable pace, within $15 \mathrm{~min}$. The test foods and reference food were served with $250 \mathrm{ml}$ water. A further $250 \mathrm{ml}$ water were given during the subsequent $2 \mathrm{~h}$. Subjects were encouraged to keep physical activity to a minimum during the testing.

\section{Blood glucose measurements}

A fasting blood sample was taken at -5 and 0 min, capillary blood was used and the reference test food was consumed immediately after this. Further blood samples were taken at $15,30,45,60,90$ and $120 \mathrm{~min}$ after starting to eat. Blood was obtained by finger-prick using the Schutz Lancetor (Chong Lap (H.K.) Co. Ltd, Kowloon, Hong Kong). Before a finger-prick, subjects were encouraged to warm their hand to increase blood flow. Fingers were not squeezed to extract blood from the fingertip in order to minimise plasma dilution. Blood glucose was measured using a HemoCue Glucose $201+$ analyser (HemoCue AB, Ängelholm, Sweden). The blood glucose meters were calibrated daily using control solutions from the manufacturer.

\section{Calculation of glycaemic index and glycaemic load}

The IAUC, ignoring the area beneath the baseline, was calculated geometrically for each food (FAO/WHO $)^{(5)}$. The mean, standard deviation and CV of the IAUC of each subject's repeated reference food were calculated. The IAUC for each test food eaten by each subject was expressed as a percentage of the mean IAUC for the reference food eaten by the same subject: GI $=($ IAUC test food/IAUC reference food $) \times 100$. The GI of each test food was taken as the mean for the whole group.

The glycaemic load (GL) of a specific serving of each food was calculated using the following equation: $\mathrm{GL}=(\mathrm{GI}$ of test food $\times$ available carbohydrate in a serving of test food $(\mathrm{g}) / 100$. The serving size of each food was taken from manufacturers' information.

\section{Statistical analysis}

Statistical analysis was performed using SAS 9.1 (SAS Institute, Inc., Cary, NC, USA). Data are presented as means, standard deviations and standard errors. Levels of 
Table 2. Composition of the ten tested foods

\begin{tabular}{|c|c|}
\hline Food & Major ingredients \\
\hline \multicolumn{2}{|l|}{ Noodles } \\
\hline 1. Jianxi rice vermicelli & Rice, water \\
\hline 2. Sau tao Beijing noodles & Wheat flour, salt, tapioca starch, water \\
\hline 3. Taiwan vermicelli & Rice, water, maize starch \\
\hline 4. Sau tao chicken-flavoured Sichuan spicy noodles & Wheat flour, salt, water \\
\hline 5. Doll fried noodles & $\begin{array}{l}\text { Wheat flour, palm oil, tapioca starch, salt, flavour enhancer, } \\
\text { acidity regulator, garlic powder, thickener }\end{array}$ \\
\hline \multicolumn{2}{|r|}{ 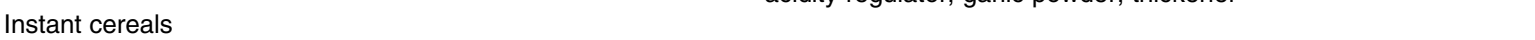 } \\
\hline 6. Quaker oatmeal (anchovies flavour) & $\begin{array}{l}\text { Quaker instant oats, maltodextrin, sugar, natural identical flavour } \\
\text { (contains fish), salt, carrots, anchovies (fish), calcium carbonate, } \\
\text { flavour enhancer, onion grit, ground ginger, leeks, ground pepper }\end{array}$ \\
\hline 7. Nestlé Vita (red date flavour, high Fe) & $\begin{array}{l}\text { Cereal base powder (whole-wheat flour, rice flour, barley } \\
\text { malt extract, sugar, corn flour, salt, calcium carbonate), sugar, } \\
\text { creamer (glucose syrup, hydrogenated vegetable oil, sodium } \\
\text { caseinate), whey powder, minerals, red date powder }(0.4 \%)\end{array}$ \\
\hline 8. Nestlé Vita (green tea flavour, high $\mathrm{Ca}$ ) & $\begin{array}{l}\text { Cereal base powder (whole wheat flour, rice flour, barley malt extract, } \\
\text { sugar, corn flour, salt, calcium carbonate), sugar, creamer } \\
\text { (glucose syrup, hydrogenated vegetable oil, sodium caseinate), } \\
\text { whey powder, minerals, tea solids }(0.36 \%)\end{array}$ \\
\hline \multicolumn{2}{|r|}{ 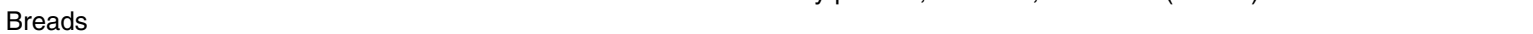 } \\
\hline 9. Garden milk bar bun & $\begin{array}{l}\text { Wheat flour, sugar, butter, egg, water, whey powder, wheat gluten, } \\
\text { yeast, salt, condensed milk }\end{array}$ \\
\hline 10. Linola seed bread & Wheat flour, water, linola seed, salt, yeast \\
\hline
\end{tabular}

inter- and intra-individual variation of the standard (glucose) tests were assessed by determining the CV\%.

Spearman's correlation coefficient $(r)$ was used to assess the relationship between GI values and nutrient content of the test foods. Statistical significance was set at $P<0 \cdot 05$.

\section{Results}

The mean intra-individual $\mathrm{CV}$ of glycaemic responses to the two $50 \mathrm{~g}$ glucose standard tests for the twenty-three subjects was $13 \%$. The inter-individual $\mathrm{CV}$ in glycaemic response to the standard tests was $25 \%$. These values are consistent with recently reported data that low mean within-subject variation (reference $\mathrm{CV}<30 \%$ ) is required for accuracy ${ }^{(13)}$.

The GI and GL values for all tested foods are given in Table 3. The GL of a specific serving of each food was calculated using the following equation: $\mathrm{GL}=(\mathrm{GI}$ test food $\times$ weight of available carbohydrate per serving of test food $(\mathrm{g}) / 100)$. GI values are given as means with their standard errors. For practical application, GI values are often grouped into categories as producing a low, medium or high glycaemic response: low $\leq 55$; medium 56-69 inclusive; high $\geq 70^{(14)}$. All bread products fell into the high-GI category, while instant cereals were medium to high. Most noodles fell into the medium-GI category, except for Doll fried noodles, which fell into the high-GI category.

There are several factors that may alter the GI of a food, including the presence of other macronutrients such as fat and protein. However, in the present study, there was no relationship between the GI value and the amount of protein per $50 \mathrm{~g}$ available carbohydrate portion (Spearman's $\rho$ $-0.394 ; P=0.130)$ and the amount of fat per $50 \mathrm{~g}$ available carbohydrate portion (Spearman's $\rho 0.333 ; P=0.173$ ). This was expected, as previous findings from Wolever et al. ${ }^{(15)}$ and Henry et al. ${ }^{(10)}$ also demonstrate that the amount of protein or fat found in commonly consumed foods does not significantly affect the glycaemic response.

\section{Discussion}

The majority of GI values of foods tested in the present study compare well with previously published values of cereals in China $^{(16)}$. For example, in healthy subjects, the GI values for instant porridge (69.4 (SD 7.2)), refined wheat bread $(87.9$ (SD 10.2)), fresh wheat noodles $(81.6$ (SD 19.1)) reported in the table of GI values published in the study of Yang et al. ${ }^{(16)}$ are similar to the values for noodles, instant cereals and breads shown in Table 3.

Rice noodles (also known as vermicelli) have been previously tested in Canadian ${ }^{(17)}$ and Vietnamese studies ${ }^{(18)}$. The GI value for Jianxi rice vermicelli tested in the present study was similar to the previously recorded value (56 $v$. 58). Taiwan vermicelli tested in the present study had a higher value and matched more closely vermicelli produced in Thailand ${ }^{(18)}$ (68 v. 61). The relatively high GI values of these noodle products might be due to their amylopectin content ${ }^{(19)}$ and difference in processing conditions and ingredients used among different countries. This therefore reconfirms the need to test food products in the country of consumption ${ }^{(9)}$.

A high GI value was obtained with Nestlé Vita instant porridge, 94 (SE 12) and 108 (SE 8) for red date and green tea flavours, respectively. Due to their high carbohydrate contents ( $80.4 \%$ dry weight) and the presence of pregelatinised starch, these foods are capable of inducing significant glycaemia in normal serving sizes ${ }^{(18)}$. With reference to the 2002 China Food Composition tables ${ }^{(20)}$, Quaker original oatmeal has a GI value of 83; however, in the present study Quaker oatmeal with anchovies showed a lower GI value to 67 (SE 5). Such differences between porridges of similar brands are most likely due to subtle differences in processing methods and/or formulations. It could be that the formation of different amounts of resistant starch could contribute to this differences in $\mathrm{GI}^{(21)}$. The GI values of Garden milk bar buns, Taiwan vermicelli, Sau tao Beijing noodles and some popular instant noodles (Sau tao chicken-flavoured noodles and Sichuan 


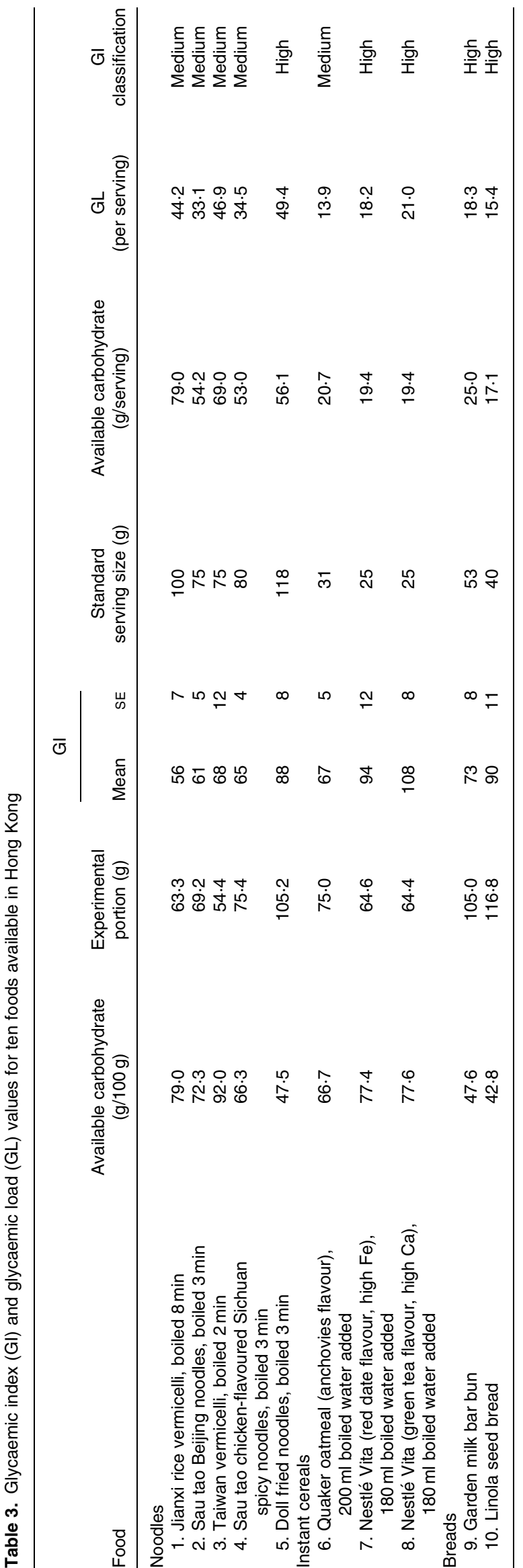

spicy noodles) and flavoured instant cereals had not been published previously. The present study further expands the GI database on Chinese foods.

The variability in published GI values for foods of similar description could be explained by real differences between the foods, and/or by variations in the methodology used. Food factors that can influence the GI include processing, preparation and cooking methods, the physical form of the food, type of sugars and starch in the food, presence of other macronutrients and anti-nutrients, and the ripeness or maturity of the food. The effects of several of these factors are illustrated by the findings of GI values of instant cereals in the present study and previous research $^{(10,21,22)}$. For example, Quaker oatmeal with anchovies and Nestlé Vita with red date or green tea have different formulations in China, Australia and Europe because manufacturers prepare and process foods, particular cereal products, in different ways ${ }^{(9)}$.

The presence of large amounts of protein or fat may significantly reduce the glycaemic response by increasing insulin secretion and slowing gastric emptying ${ }^{(23,24)}$. However, in the present study, the effect of protein and fat was not observed across the group of foods. As expected, there was no association with fat or protein content per $50 \mathrm{~g}$ available carbohydrate portion of the test foods. This is consistent with previous findings of Wolever et al. ${ }^{(15)}$ and Henry et al. ${ }^{(10)}$ that demonstrated that the amount of protein or fat in commonly consumed foods does not significantly affect the glycaemic response.

A limitation of the present study is the use of varying methodology by different laboratories for the measurement and calculation of GI that also contributes to the variation seen in published GI values. Although there is a recommended standard protocol for the determination of $\mathrm{GI}^{(5)}$, there is scope within this for slight variations, and an interlaboratory study has found that differences in GI of up to 18 units may in part be owing to methodological factors rather than real differences between the foods ${ }^{(25)}$. Such variations include the use of venous rather than capillary blood, the use of different food composition tables to calculate the required portion size, and the method used for determining the $\mathrm{AUC}^{(21)}$.

In conclusion, the present study has provided reliable values of GI and GL for foods commonly consumed in Hong Kong. It has also reconfirmed some of the multiple factors affecting the GI of a food, which illustrates the importance of measuring GI values of foods in the country of consumption rather than applying values from foods of a similar description or brand. In addition to this, our dataset provides GI values of several foods previously untested. Knowledge of the GI values of Chinese foods may assist in the development of better dietary advice for individuals with diabetes and obesity and will aid further research into the application of GI.

\section{Acknowledgements}

We would like to thank Science International Corporation for their support and supply of the HemoCue Glucose $201+$ analyser. The study was supported by the Centre for Nutritional Studies.

L. L. and G. L. undertook the assessment and data collection. D. C. carried out statistical analysis of the study. K. Y L. analysed the data and wrote the manuscript with input 
from all authors. R. C., J. W., H. J. L. and C. J. K. H. participated in the conception and design of the study.

There is no conflict of interest.

\section{References}

1. Jenkins DJA, Wolever TMS, Taylor RH, et al. (1981) Glycemic index of foods: a physiological basis for carbohydrate exchange. Am J Clin Nutr 34, 362-366.

2. Aziz A (2009) The glycemic index: methodological aspects related to the interpretation of health effects and to regulatory labeling. J AOAC 92, 879-887.

3. Radulian G, Rusu E, Dragomir A, et al. (2009) Metabolic effects of low GI diets. Nutr J 8, 5 (epublication 29 January 2009).

4. Bjorck I, Granfeldt Y, Liljeberg H, et al. (1994) Food properties affecting the digestion and absorption of carbohydrates. Am J Clin Nutr 59, S699-S705.

5. Food and Agriculture Organization \& World Health Organization (1998) Carbohydrates in Human Nutrition. Report of a Joint FAO/WHO Expert Consultation. FAO Food and Nutrition Paper no. 66. Rome: FAO.

6. Liese AD, Schulz M, Fang F, et al. (2005) Dietary glycemic index and glycemic load, carbohydrate and fiber intake, and measures of insulin sensitivity, secretion, and adiposity in the Insulin Resistance Atherosclerosis Study. Diabetes Care 28, $2832-2838$.

7. Hodge AM, English DR, O'Dea K, et al. (2004) Glycemic index and dietary fiber and the risk of type 2 diabetes. Diabetes Care 27, 2701-2706.

8. Barclay AW, Flood WM, Rochtchina E, et al. (2007) Glycemic index, dietary fiber, and risk of type 2 diabetes in a cohort of older Australians. Diabetes Care 30, 2811-2813.

9. Atkinson FS, Foster-Powell K \& Brand-Miller JC (2008) International tables of glycemic index and glycemic load values: 2008. Diabetes Care 31, 2281-2283.

10. Henry CJK, Lightowler HJ, Strik CM, et al. (2005) Glycaemic index and glycaemic load values of commercially available products in the UK. Br J Nutr 94, 922-930.
11. Wolever TMS, Jenkins DJA, Jenkins AL, et al. (1991) The glycemic index: methodology and clinical implications. Am J Clin Nutr 54, 846-854.

12. Brouns F, Bjorck I, Frayn KN, et al. (2005) Glycaemic index methodology. Nutr Res Rev 18, 145-171.

13. Wolever TMS, Brand-Miller JC, Abernethy J, et al. (2008) Measuring the glycemic index of foods: interlaboratory study. Am J Clin Nutr 87, Suppl., 247S-257S.

14. Brand-Miller J, Foster-Powell K \& Colagiuri S (2003) The New Glucose Revolution. New York: Marlowe and Company.

15. Wolever TMS, Katzmanrelle L, Jenkins AL, et al. (1994) Glycemic index of 102 complex carbohydrate foods in patients with diabetes. Nutr Res 14, 651-669.

16. Yang YX, Wang HW, Cui HM, et al. (2006) Glycemic index of cereals and tubers produced in China. World J Gastroenterol 12, 3430-3433.

17. Foster-Powell K \& Miller JB (1995) International tables of glycemic index. Am J Clin Nutr 62, 871S-890S.

18. Chan HMS, Brand-Miller J, Holt SHA, et al. (2001) The glycaemic index values of Vietnamese foods. Eur J Clin Nutr 55, 1076-1083.

19. Wolever TMS (1990) The glycemic index. World Rev Nutr Diet 62, 120-185.

20. Yang Y, Wang G \& Pan X (2002) China Food Composition 2002. Beijing: Peking University Medical Press.

21. Aston LM, Gambell J, Lee DM, et al. (2008) Determination of the glycaemic index of various staple carbohydrate-rich foods in the UK diet. Eur J Clin Nutr 62, 279-285.

22. Henry CJK, Lightowler HJ, Dodwell LM, et al. (2007) Glycaemic index and glycaemic load values of cereal products and weight-management meals available in the UK. Br J Nutr 98, 147-153.

23. Collier G, McLean A \& O'Dea K (1984) Effect of co-ingestion of fat on the metabolic responses to slowly and rapidly absorbed carbohydrates. Diabetologia 26, 50-54.

24. Nutall FQ, Mooradian AD, Gannon MC, et al. (1984) Effect of protein ingestion on the glucose and insulin response to a standardized oral glucose load. Diabetes Care 7, 465-470.

25. Wolever TMS (2003) Carbohydrate and the regulation of blood glucose and metabolism. Nutr Res 61, S40-S48. 